\title{
The Effect of Botulinum Toxin on an Iatrogenic Sialo-Cutaneous Fistula
}

\author{
Seung Eun Hong, \\ Jung Woo Kwon, \\ So Ra Kang, \\ Bo Young Park
}

Department of Plastic and Reconstructive Surgery, Ewha Womans University Mokdong Hospital, Ewha Womans University School of Medicine, Seoul, Korea

No potential conflict of interest relevant to this article was reported.

\begin{abstract}
A sialo-cutaneous fistula is a communication between the skin and a salivary gland or duct discharging saliva. Trauma and iatrogenic complications are the most common causes of this condition. Treatments include aspiration, compression, and the administration of systemic anticholinergics; however, their effects are transient and unsatisfactory in most cases. We had a case of a patient who developed an iatrogenic sialo-cutaneous fistula after wide excision of squamous cell carcinoma in the parotid region that was not treated with conventional management, but instead completely resolved with the injection of botulinum toxin. Based on our experience, we recommend the injection of botulinum toxin into the salivary glands, especially the parotid gland, as a conservative treatment option for sialo-cutaneous fistula.
\end{abstract}

Keywords: Salivary gland fistula / Botulinum toxins / Squamous cell carcinoma

\section{INTRODUCTION}

A sialo-cutaneous fistula is defined as a communication between the skin and a salivary gland resulting in the discharge of saliva from the skin [1]. Common causes of sialo-cutaneous fistula are trauma, a complication from sialolithiasis, and an iatrogenic complication from surgery, usually related to the parotid gland. Both surgical and non-surgical approaches have been accepted as treatment modalities, and recently, the injection of botulinum toxin has been reported to be a successful treatment [2]. The major secretomotor fibers to the parotid gland are cholinergic parasympathetic, which are susceptible to inhibition by botulinum toxin [3]. The local injection of type A botulinum toxin represents a new and effective treatment for sialo-cutaneous fistulas and is less in-

Correspondence: Bo Young Park

Department of Plastic and Reconstructive Surgery, Ewha Womans University Mokdong Hospital, Ewha Womans University School of Medicine, 1071 Anyangcheon-ro, Yangcheon-gu, Seoul 07985, Korea

E-mail: byps@icould.com

Received June 14, 2016 / Revised December 13, 2016 / Accepted December 13, 2016 vasive, stressful, and lengthy than conventional methods. We report a case in which an iatrogenic sialo-cutaneous fistula in the preauricular area after skin cancer removal was successfully treated with the injection of type A botulinum toxin.

\section{CASE REPORT}

An 80-year-old male was referred to us with squamous cell carcinoma of the left pre-auricular area that was confirmed through incisional biopsy (Fig. 1A). The patient was suffering from occasional bleeding, discharge, and repeated ulceration of the lesion. We performed computed tomography of the head and neck region before surgery, which showed no evidence of invasion. We performed wide excision of the tumor with a $0.5 \mathrm{~cm}$ lateral margin throughout. We obtained a safe margin of depth without parotid gland injury even though the tumor bed was close to the parotid gland fascia (Fig. 1B). However, we could not completely rule out micro-injuries to the parotid gland fascia although we reserved parotid gland fascia. After wide excision, the skin defect was covered with a $5 \times 6 \mathrm{~cm}$ full thickness skin graft from the left 

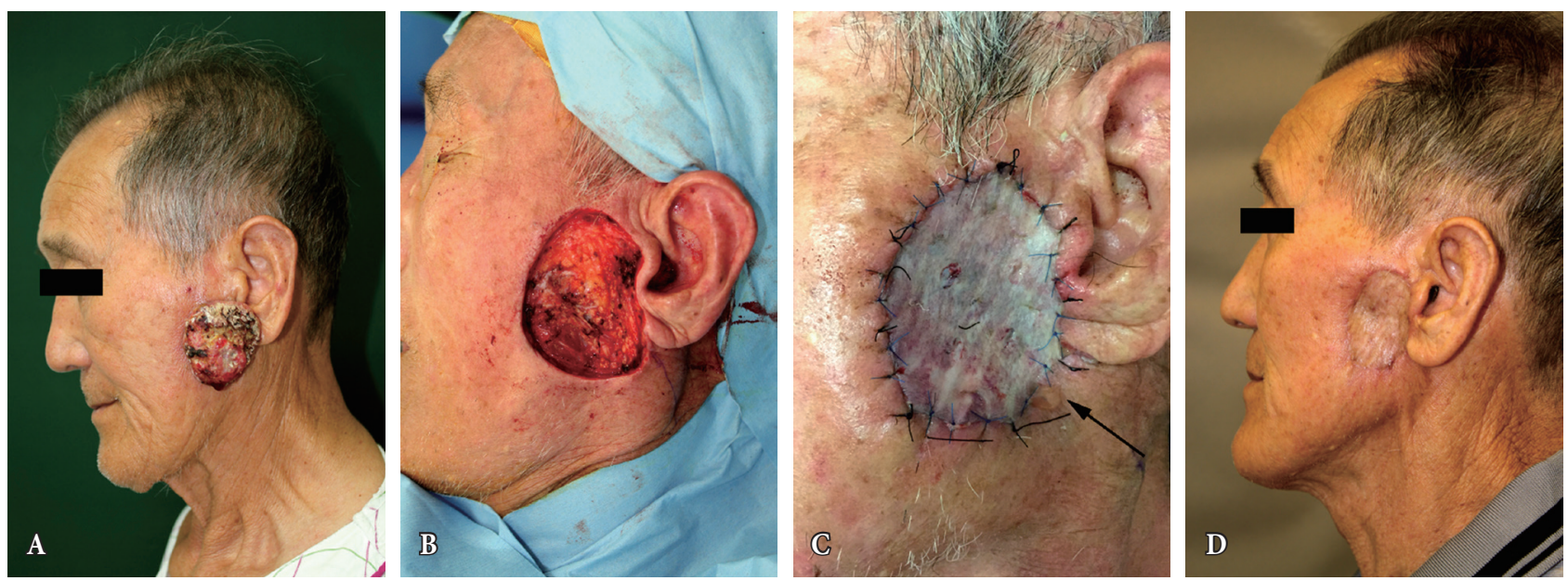

Fig. 1. (A) An 80-year-old male with a protruding mass on the left pre-auricular area. Punch biopsy results were consistent with squamous cell carcinoma. (B) After cancer resection. Parotid fascia was grossly intact. (C) On the fifth postoperative day, the patient complained of sialorrhea from the wound. Salivary leakage was found from the slit incision site of the full thickness skin graft (arrow). Sialorrhea from the parotid gland. We injected Botulinum Toxin Type A 50 unit into the parotid gland tissue. (D) Four weeks after surgery.

inguinal area. The usual compression dressing with a tie-over suture was applied to maintain the skin graft.

On the fifth postoperative day, we discovered exudate from the base of the skin graft through the slit incision site (Fig. 1C). The dressing materials were wet and the discharge from the operative site increased during meals. The amount of discharge was approximately $50 \mathrm{~mL}$, and its amylase level was 200,000 unit/L. Accordingly, we confirmed the exudate was salivary discharge and he was diagnosed with a sialocutaneous fistula.

We decided to use botulinum toxin type A injection because of concern for graft failure due to increased sialorrhea that was not controlled by conventional compression dressings. Using a tuberculin syringe, we administered a transcutaneous injection of 25 IU of the toxin at a point $0.5 \mathrm{~cm}$ above the site of the fistula at the surgical incision line and administered another 25 IU injection $0.5 \mathrm{~cm}$ anterior from that point. The injections were performed only for the superficial parotid gland in order to avoid post injection facial nerve paralysis. The discharge draining from the sialocutaneous fistula was considerably reduced after the second day of injection. The patient fully recovered within a week without any complications such as graft shearing or failure (Fig. 1D).

\section{DISCUSSION}

Sialo-cutaneous fistula is one of the most common complications of post-trauma injury and head and neck surgery. There has not been a consensus on the appropriate treatment of this condition, which range from conservative methods such as needle aspiration, pressure dressings, and anti-sialogogue therapy, to radiotherapy and surgical approaches such as duct repair, diversion, ligation, drainage systems, and even parotidectomy [4]. Most cases are not self-limiting and respond poorly to several weeks of conservative therapy. Pharmacological options are limited, frequently unsatisfactory, and associated with considerable side effects. In addition, there are considerable economic costs of postoperative complications and their appropriate management approaches which often require a longer hospital stay [5].

The parotid gland receives both sensory and autonomic innervation. Sensory innervation is supplied by the auriculotemporal nerve, a branch of the mandibular nerve. The autonomic innervation controls the rate of saliva production. Preganglionic parasympathetic fibers leave the brain stem from the inferior salivatory nucleus in the glossopharyngeal nerve and then run along its tympanic branch followed by the lesser petrosal branch into the otic ganglion. There, they synapse with postganglionic fibers 
which reach the gland by hitch-hiking via the auriculotemporal nerve, a branch of the mandibular nerve [6]. The major secretomotor fibers to the salivary gland are cholinergic parasympathetics, which can be modulated by botulinum toxin.

Botulinum toxin has transient effects but has many advantages including the fact that it is non-destructive, dose-dependent, has good target area localization, and has minimal systemic side effects [7]. Botulinum toxin causes chemical denervation by blocking the release of the neurotransmitter acetylcholine at cholinergic nerve terminals. Since acetylcholine is the major neurotransmitter of the peripheral parasympathetic nervous system, it is possible to modulate parasympathetic stimulation by botulinum toxin [8].

Recently, botulinum toxin has been used in some autonomic diseases such as achalasia, hyperhidrosis, and Frey's syndrome [9]. There have been reports of using botulinum toxin to treat cases of parotid sialocele resistant to conventional modes of treatment. Dessy et al. [10] reported the effectiveness of botulinum toxin injection for a parotid fistula after face-lift surgery. There have been described numerous methods for administering botulinum toxin injection to treat a fistula, but no consensus currently exists regarding the amount, approach or site to prevent paralysis of the facial nerve [11].

Considering the 18,938 face-lift procedures performed in South Korea in 2014, the incidence of sialo-cutaneous fistula could be substantial and surgeons should consider the administration of botulinum toxin as a non-invasive and effective treatment modality [12]. The injection of botulinum toxin is a highly effective, safe, and non-invasive therapy for this condition [13].

Based on our experience, we recommend the injection of botulinum toxin as a conservative treatment option for parotid sialocutaneous fistula. Botulinum toxin avoids the side effects caused by systemic anticholinergic drugs and is cost-effective. However, it is essential that the accurate anatomical landmarks are recognized by a trained injector before proceeding.

\section{REFERENCES}

1. Feingold RS. Parotid salivary gland fistula following rhytidectomy. Plast Reconstr Surg 1998;101:245.

2. McKinney P, Zuckerbraun BS, Smith JW, Kawamoto HK, Griffith BH. Management of parotid leakage following rhytidectomy. Plast Reconstr Surg 1996;98:795-7.

3. Tugnoli V, Eleopra R, Montecucco C, De Grandis D. The therapeutic use of botulinum toxin. Expert Opin Investig Drugs 1997;6:1383-94.

4. Parekh D, Glezerson G, Stewart M, Esser J, Lawson HH. Post-traumatic parotid fistulae and sialoceles: a prospective study of conservative management in 51 cases. Ann Surg 1989;209:105-11.

5. Lim YC, Choi EC. Treatment of an acute salivary fistula after parotid surgery: botulinum toxin type A injection as primary treatment. Eur Arch Otorhinolaryngol 2008;265:243-5.

6. Proctor GB. The physiology of salivary secretion. Periodontol 2000 2016;70:11-25.

7. Hallett M. One man's poison: clinical applications of botulinum toxin. N Engl J Med 1999;341:118-20.

8. Marchese-Ragona R, De Filippis C, Staffieri A, Restivo DA, Restino DA. Parotid gland fistula: treatment with botulinum toxin. Plast Reconstr Surg 2001;107:886-7.

9. Marchese-Ragona R, Marioni G, Restivo DA, Staffieri A. The role of botulinum toxin in postparotidectomy fistula treatment: a technical note. Am J Otolaryngol 2006;27:221-4.

10. Dessy LA, Mazzocchi M, Monarca C, Onesti MG, Scuderi N. Combined transdermal scopolamine and botulinum toxin $\mathrm{A}$ to treat a parotid fistula after a face-lift in a patient with siliconomas. Int J Oral MaxillofacSurg 2007;36:949-52.

11. Melville JC, Stackowicz DJ, Jundt JS, Shum JW. Use of Botox (OnabotulinumtoxinA) for the treatment of parotid sialocele and fistula after extirpation of buccal squamous cell carcinoma with immediate reconstruction using microvascular free flap: a report of 3 cases. J Oral Maxillofac Surg 2016;74:1678-86.

12. International Society of Aesthetic Plastic Surgery (SAPS) glocal statistics. ISAPS International Survey on Aesthetic/Cosmetic Procedures Performed in 2014 [Internet]. Hanover: International Society of Aesthetic Plastic Surgery; [cited 2016 Feb 2]. Available from: http://www. isaps.org/Media/Default/global-statistics/2015\%20ISAPS\%20Results. pdf.

13. Vargas H, Galati LT, Parnes SM. A pilot study evaluating the treatment of postparotidectomy sialoceles with botulinum toxin type A. Arch Otolaryngol Head Neck Surg 2000;126:421-4. 